\title{
POINTWISE MULTIPLIERS OF WEIGHTED BMO SPACES
}

\author{
KÔZÔ YABUTA \\ (Communicated by J. Marshall Ash) \\ Dedicated to Professor Takeshi Kotake on his sixtieth birthday
}

\begin{abstract}
In a recent paper by S. Bloom (Pointwise multipliers of weighted BMO spaces, Proc. Amer. Math. Soc. 105 (1989), 950-960), there are some inaccuracies. In this note, we give a counterexample to his "theorem" and a corrected form with proof under a suitable condition on weights. We also give some remarks and examples.
\end{abstract}

\section{INTRODUCTION}

Throughout this note, $w$ will be a doubling measure on $\mathbb{R}^{n}$, i.e., there is a nonnegative function $w(x)$ satisfying the property

$$
w(J)=\int_{J} w(x) d x \leq C w(I),
$$

whenever cubes $I, J$ satisfy $I \subset J$ and $|J| \leq 2^{n}|I|$. Furthermore, we always assume

$$
w(x) \geq C_{I}>0, \quad x \in I \text { for all cubes } I .
$$

(This is equivalent to saying that $f \in L_{\text {loc }}^{1}(w(x) d x)$ implies $f \in L_{\text {loc }}^{1}\left(\mathbb{R}^{n}\right)$.) $\mathbb{D}$ will denote the set of all dyadic cubes, i.e.,

$$
\mathbb{D}=\left\{\text { cubes of the form } \prod_{j=1}^{n}\left[k_{j} 2^{l},\left(k_{j}+1\right) 2^{l}\right) ; k \in \mathbb{Z}^{n}, l \in \mathbb{Z}\right\} \text {. }
$$

For a cube $I$ and an $f \in L_{\text {loc }}^{1}\left(\mathbb{R}^{n}\right), \quad f_{I}$ is defined by $f_{I}=\frac{1}{|I|} \int_{I} f(x) d x$. The dyadic maximal operator is

$$
M_{d}^{*} f(x)=\sup \left\{\left|f_{I}\right| ; x \in I, I \in \mathbb{D}\right\} .
$$

A locally integrable function $b$ is said to belong to $\mathrm{BMO}_{w}$ if

$$
\|b\|_{\mathrm{BMO}_{w}}=\sup \left\{\frac{1}{w(I)} \int_{I}\left|b(x)-b_{I}\right| d x\right\}<+\infty,
$$

Received by the editors January 9, 1991 and, in revised form, June 25, 1991.

1991 Mathematics Subject Classification. Primary 42B15; Secondary 42B30, 42B25. spaces.

Key words and phrases. Pointwise multipliers, weighted BMO, BMO, dyadic, weighted Hardy 
where the supremum is taken over all cubes $I$ with sides parallel to the coordinate axes.

A locally integrable function $b$ is said to belong to $\mathrm{BMO}_{w, d}$ if

$$
\|b\|_{\mathrm{BMO}_{w, d}}=\sup _{I \in \mathbb{D}}\left\{\frac{1}{w(I)} \int_{I}\left|b(x)-b_{I}\right| d x\right\}<+\infty .
$$

A $w$-atom is a function supported in a cube $I$ with $\int a(x) d x=0$ and $\|a\|_{\infty} \leq$ $1 / w(I) . f \in H_{w}^{1}$ if there exist $w$-atoms $\left\{a_{j}\right\}$ and a sequence $\left\{\lambda_{j}\right\} \in l^{1}$ such that $f=\sum \lambda_{j} a_{j}$ in $L^{1}(w)$, and we set

$$
\|a\|_{H_{w}^{1}}=\inf \left\{\sum\left|\lambda_{j}\right| ; f=\sum \lambda_{j} a_{j}\right\} .
$$

Similarly, a $(w, d)$-atom is a function supported in a cube $I \in \mathbb{D}$ with $\int a(x) d x=0$ and $\|a\|_{\infty} \leq 1 / w(I) . f \in H_{w, d}^{1}$ if there exist $(w, d)$-atoms $\left\{a_{j}\right\}$ and a sequence $\left\{\lambda_{j}\right\} \in l^{1}$ such that $f=\sum \lambda_{j} a_{j}$ in $L^{1}(w)$, and we set

$$
\|a\|_{H_{w, d}^{1}}=\inf \left\{\sum\left|\lambda_{j}\right| ; f=\sum \lambda_{j} a_{j}, a_{j}:(w, d) \text {-atoms }\right\} .
$$

For our weight $w$, we have the following (cf. [6]).

Proposition 1. $f \in L_{\mathrm{loc}}^{1}\left(\mathbb{R}^{n}\right)$ belongs to $H_{w, d}^{1}$ if and only if $M_{d}^{*} f \in L^{1}(w)$, and $\left\|M_{d}^{*} f\right\|_{L^{1}(w)}$ is equivalent to $\|f\|_{H_{w, d}^{1}}$.

We note that this proposition does not hold for general doubling measures, even if $w \in A_{p}\left(\mathbb{R}^{n}\right) \quad(1<p$ and $p n<n+1)$ (hence $w \in D_{p n}$ in the sense of Strömberg-Torchinsky [5]) and the convergence of atoms in the definition of $H_{w}^{1}$ is taken in the sense of distributions, see Example 1 in $\S 3$. Here $A_{p}$ is Muckenhoupt's weight class.

Next, modifying the proof of the $H^{1}$-BMO duality by Coifman and Weiss [2, pp. 631-634], one can show

Proposition 2. Every $b \in \mathrm{BMO}_{w, d}$ determines a bounded linear functional on $H_{w, d}^{1}$ and the dual form is given by

$$
\langle f, b\rangle=\sum \lambda_{j} \int a_{j}(x) b(x) d x,
$$

where $\sum \lambda_{j} a_{j}$ is any atomic decomposition of $f$. Furthermore

$$
\langle f, b\rangle=\int f(x) b(x) d x
$$

in the case where $f$ is a finite linear combination of $(w, d)$-atoms and $b \in$ $\mathrm{BMO}_{w, d}$, and in the case where $f \in H_{w, d}^{1}$ and $b / w \in L^{\infty}\left(\mathbb{R}^{n}\right)$.

Now, as for pointwise multipliers of $H_{w, d}^{1}$ we have

Theorem 1. $\phi(x) f(x) \in H_{w, d}^{1}$ for every $f \in H_{w, d}^{1}$ if and only if

$$
\phi \in L^{\infty}
$$

and

$$
\int_{I}\left|\phi(x)-\phi_{I}\right| d x \leq C w(I) / \int_{I^{c}} \frac{w(x) d x}{\left|x-x_{I}\right|^{n}} \quad \text { for all } I \in \mathbb{D},
$$

where $x_{I}$ is the center of the cube $I$. 
The proof of Theorem 1.4 in Bloom [1] is valid in this case, in virtue of Proposition 2. His statement for $\mathrm{BMO}_{w}$ in his Theorem 1.7, however, is not correct; a counterexample will be given later. His Theorem 1.4 should read as follows.

Theorem 2. Suppose $\int \frac{w(x) d x}{1+|x|^{n}}<+\infty$. Then $\phi(x) b(x) \in \mathrm{BMO}_{w, d}$ for every $b \in \mathrm{BMO}_{w, d}$ if and only if

$$
\phi \in L^{\infty}
$$

and

$$
\int_{I}\left|\phi(x)-\phi_{I}\right| d x \leq C w(I) /\left(1+\int_{I^{c}} \frac{w(x) d x}{\left|x-x_{I}\right|^{n}}\right) \text { for all } I \in \mathbb{D} \text {. }
$$

Remark. (1) $+\left(2^{\prime}\right)$ is equivalent to $\left(1^{\prime}\right) \phi \in L^{\infty} \cap \mathrm{BMO}_{w, d}+(2)$.

\section{Proof of Theorem 2}

For the proof we prepare several lemmas. Since $M_{d}^{*} f \leq C\left(1+|x|^{n}\right)^{-1}$ for every bounded function $f$ with compact support, we have by Proposition 1

Lemma 1. Suppose $\int \frac{w(x) d x}{1+|x|^{n}}<+\infty$. Then every bounded function with compact support belongs to $H_{w, d}^{1}$.

Lemma 2. Let $w(x)$ be as in Lemma 1 . Then if $b \in \mathrm{BMO}_{w, d}$ we have for any cube $I \in \mathbb{D}$

$$
\lim _{j \rightarrow \infty} \frac{1}{\left|I^{j}\right|} \int_{I^{j}} b(x) d x=b_{\infty, I} \text { and } \lim _{j \rightarrow \infty} \frac{1}{\left|I^{j}\right|}\left\langle\chi_{I^{j}}, b(x)\right\rangle=b_{\infty \infty, I}
$$

exist, where $I^{1}=I$ and $I^{j}$ is the unique cube $J \in \mathbb{D}$ with $I \subset J$ and $|J|=$ $2^{n}\left|I^{j-1}\right|, j=2,3, \ldots$.

Proof (by A. Uchiyama). We may assume $I$ is of the form $\left[0,2^{m}\right)^{n}$. Let $I \subset J \in \mathbb{D}$. Then

$$
\begin{aligned}
\left|\frac{1}{|J|} \int_{J} b d y-\frac{1}{\left|J^{2}\right|} \int_{J^{2}} b d y\right| & =2^{n} \cdot \frac{1}{\left|J^{2}\right|} \int_{J}\left|b-b_{J^{2}}\right| d y \\
& \leq C \frac{1}{\left|J^{2}\right|} \int_{J^{2}}\left|b-b_{J^{2}}\right| d y \leq C\|b\|_{\mathrm{BMO}_{w, d}} \frac{w\left(J^{2}\right)}{\left|J^{2}\right|} \\
& \leq C\|b\|_{\mathrm{BMO}_{w, d}} \int_{J^{2} \backslash J} \frac{w(x)}{|x|^{n}} d x .
\end{aligned}
$$

Hence

$$
\sum_{j=2}^{\infty}\left|\frac{1}{\left|I^{j}\right|} \int_{I^{j}} b d y-\frac{1}{\left|I^{j-1}\right|} \int_{I^{j-1}} b d y\right| \leq C\|b\|_{\mathrm{BMO}_{w, d}} \int_{\mathbb{R}^{n}} \frac{w(x)}{(1+|x|)^{n}} d x<+\infty .
$$

Thus, $b_{\infty, I}$ exists.

Next, since $\int\left(\frac{1}{|J|} \chi_{J}-\frac{1}{\left|J^{2}\right|} \chi_{J^{2}}\right) d x=0$, supp $\frac{1}{|J|} \chi_{J}-\frac{1}{\left|J^{2}\right|} \chi_{J^{2}} \subset J^{2}$, we see that $|J|\left(\frac{1}{|J|} \chi_{J}-\frac{1}{\left|J^{2}\right|} \chi_{J^{2}}\right) / w\left(J^{2}\right)$ is a $(w, d)$-atom, and so by Proposition 2

$$
\left|\left\langle\frac{1}{|J|} \chi_{J}, b\right\rangle-\left\langle\frac{1}{\left|J^{2}\right|} \chi_{J^{2}}, b\right\rangle\right| \leq C \frac{w\left(J^{2}\right)}{|J|}\|b\|_{\mathrm{BMO}_{w, d}} .
$$

Thus, as before, we see that $b_{\infty \infty, I}$ exists. Q.E.D. 
Now there are $2^{n}$ quadrants $K(j)$ with respect to $\mathbb{D}$, such that each $I \in \mathbb{D}$ is contained uniquely in one of them. For $b \in \mathrm{BMO}_{w, d}$ let

$$
b_{\infty, K(j)}=b_{\infty, I} \text { and } b_{\infty \infty, K(j)}=b_{\infty \infty, I},
$$

where $I \subset K(j)$. Note that these are independent of the choice of $I \subset K(j)$. Set

$$
b_{\infty}=\sum_{j=1}^{2^{n}} b_{\infty, K(j)} \chi_{K(j)} \quad \text { and } \quad b_{\infty \infty}=\sum_{j=1}^{2^{n}} b_{\infty \infty, K(j)} \chi_{K(j)} .
$$

The following lemma is proved easily.

Lemma 3. Let $w$ be as in Lemma 1. Suppose $b_{j} \in \mathrm{BMO}_{w, d}$ and $b_{j} \rightarrow b$ in $\mathrm{BMO}_{w, d}$. Then

$$
\lim _{j \rightarrow \infty}\left(b_{j}\right)_{\infty \infty}=b_{\infty \infty},
$$

and there exists a subsequence $\left\{j_{l}\right\}$ such that for almost all $y \in \mathbb{R}^{n}$

$$
\lim _{l \rightarrow \infty}\left(b_{j_{l}}(y)-\left(b_{j_{l}}\right)_{\infty}\right)=b(y)-b_{\infty} .
$$

Using this and the fact that $b-b_{\infty}+b_{\infty \infty}=0$ if $b$ is constant on each quadrant, we have by the closed graph theorem

Lemma 4. Let $w$ be as in Lemma 1. Suppose $\phi\left(b-b_{\infty}+b_{\infty \infty}\right) \in \mathrm{BMO}_{w, d}$ for every $b \in \mathrm{BMO}_{w, d}$. Then

$$
\left\|\phi\left(b-b_{\infty}+b_{\infty \infty}\right)\right\|_{\mathrm{BMO}_{w, d}} \leq C\|b\|_{\mathrm{BMO}_{w, d}}
$$

holds.

Using Lemma 1 and Proposition 2, we have

Lemma 5. Let $w$ be as in Lemma 1. Suppose $\phi f \in H_{w, d}^{1}$ for every $f \in H_{w, d}^{1}$. Then for any $I \in \mathbb{D}$

$$
\langle\phi f, b\rangle=\int f \phi\left(b-b_{I}+\left\langle\frac{1}{|I|} \chi_{I}, b\right\rangle\right) d x
$$

for all $b \in \mathrm{BMO}_{w, d}$ and all $f \in H_{w, d}^{1}$, whose atoms are of finite number and are contained in $I$.

Using Lemma 3, Lemma 5, and Proposition 2, we have

Lemma 6. Let $w$ and $\phi$ be as in Lemma 5. Then, if $b \in \mathrm{BMO}_{u, d}$, it follows that

$$
\begin{gathered}
\phi\left(b-b_{\infty}+b_{\infty \infty}\right) \in \mathrm{BMO}_{u, d}, \\
\left\|\phi\left(b-b_{\infty}+b_{\infty \infty}\right)\right\|_{\mathrm{BMO}_{w, d}} \leq\left\|M_{\phi}\right\|_{H_{w, d}^{\prime}, H_{w, d}^{\prime}}^{\prime}\|b\|_{\mathrm{BMO}_{w, d}},
\end{gathered}
$$

where $M_{\phi}$ is the multiplication operator by $\phi$.

Now using Proposition 2, Lemma 4, and Lemma 1, one can show 
Lemma 7. Let $w$ be as in Lemma 1. Then, if there exist $\phi \in L^{\infty}\left(\mathbb{R}^{n}\right)$ and $\phi\left(b-b_{\infty}+b_{\infty \infty}\right) \in \mathrm{BMO}_{w, d}$ for every $b \in \mathrm{BMO}_{w, d}$, it follows that $\phi f \in H_{w, d}^{1}$ for every $f \in H_{w, d}^{1}$.

The following lemma can be proved in the same way as in the proof of Theorem 1(1), using Proposition 2 and Lemma 1.

Lemma 8. Let $w(x) d x$ be a doubling measure satisfying (*). Then, if $\phi b \in$ $\mathrm{BMO}_{w, d}$ for every $b \in \mathrm{BMO}_{w, d}$, it follows that $\phi \in L^{\infty}\left(\mathbb{R}^{n}\right)$.

Now we are in a position to prove Theorem 2.

The proof of Theorem 2. Suppose $\phi b \in \mathrm{BMO}_{w, d}$ for every $b \in \mathrm{BMO}_{w, d}$. Then, since $1 \in \mathrm{BMO}_{w, d}$, we have $\phi \in \mathrm{BMO}_{w, d}$. Next, using Lemmas 8 and 7, we see that $\phi f \in H_{w, d}^{1}$ for every $f \in H_{w, d}^{1}$. Hence, by Theorem 1, $\phi$ satisfies (1) and (2). Thus $\phi$ satisfies (1) and $\left(2^{\prime}\right)$.

Next, suppose $\phi$ satisfies (1) and (2'), and let $b \in \mathrm{BMO}_{w, d}$. Then $\phi \in$ $\mathrm{BMO}_{w, d}$, and by Theorem $1, \phi f \in H_{w, d}^{1}$ for every $f \in H_{w, d}^{1}$. Thus by Lemma $6 \phi\left(b-b_{\infty}+b_{\infty \infty}\right) \in \mathrm{BMO}_{w, d}$. Since $b_{\infty}$ and $b_{\infty \infty}$ are constant on each quadrant and $\phi \in \mathrm{BMO}_{w, d}$, it follows that $\phi b \in \mathrm{BMO}_{w, d}$. Q.E.D.

As an easy consequence we have

Proposition 3. Let $w(x)$ be as in Theorem 2. Then, if $\phi \in L^{\infty}\left(\mathbb{R}^{n}\right)$ satisfies $\left(2^{\prime}\right)$ for all cubes $I$, it follows that $\phi(x) b(x) \in \mathrm{BMO}_{w}$ for every $b \in \mathrm{BMO}_{w}$.

Proof. For $t \in \mathbb{R}^{n}$, let $\mathbb{D}_{t}=\{I=J+t ; J \in \mathbb{D}\}$ and introduce $H_{w, d, t}^{1}$ and BMO $_{w, d, t}$ by replacing $\mathbb{D}$ by $\mathbb{D}_{t}$. Then, Propositions 1 and 2 , Theorems 1 and 2, and Lemmas 1 through 8 hold. Furthermore, the equivalence in Proposition 1 is independent of $t \in \mathbb{R}^{n}$, and if $\phi$ satisfies (1) and ( $\left.2^{\prime}\right)$, the operator norm $\left\|M_{\phi}\right\|_{H_{w, d, t}^{1}, H_{w, d, t}^{1}}$ is bounded by a constant independent of $t \in \mathbb{R}^{n}$. Now, let $I$ be a cube. Then, there exist $t \in \mathbb{R}^{n}$ and $J, K \in \mathbb{D}_{t}$ such that $J \subset I \subset J^{2}$, $|K|=1, \operatorname{dist}(0, K)=0$, and $K \subset J^{k}$ for some $k \in \mathbb{N}$. We have by Lemma 6

$$
\begin{aligned}
& \frac{1}{w(I)} \int_{I}\left|\phi b-(\phi b)_{I}\right| d x \leq \frac{C}{w\left(J^{2}\right)} \int_{J^{2}}\left|\phi b-(\phi b)_{J^{2}}\right| d x \\
& \quad \leq C\left\|M_{\phi}\right\|_{H_{w, d, t}^{1}, H_{w, d, t}^{1}}\|b\|_{\mathrm{BMO}_{w, d, t}}+C\left(\left|b_{\infty, J^{2}}\right|+\left|b_{\infty \infty, J^{2}}\right|\right)\|\phi\|_{\mathrm{BMO}_{w, d, t}} .
\end{aligned}
$$

Note that $b_{\infty, J^{2}}=b_{\infty, K}$ and $b_{\infty \infty, J^{2}}=b_{\infty \infty, K}$. Hence, as in the proof of Lemma 2 we have

$$
\begin{aligned}
\left|b_{\infty, J^{2}}\right| & \leq C\|b\|_{\mathrm{BMO}_{w, d, t}} \int_{K^{c}} \frac{w(x)}{\left|x-x_{K}\right|^{n}} d x+\left|b_{K}\right| \\
& \leq C\|b\|_{\mathrm{BMO}_{w}} \int \frac{w(x)}{(1+|x|)^{n}} d x+C \int_{|x| \leq 1}|b(x)| d x .
\end{aligned}
$$

Since $\left\|\chi_{K}\right\|_{H_{w, d, t}^{1}} \leq C\left\|M_{d, t}^{*} \chi_{K}\right\|_{L^{\prime}(w)} \leq C \int \frac{w(x) d x}{(1+|x|)^{n}}$, we have as above

$$
\left|b_{\infty \infty, J^{2}}\right| \leq C\|b\|_{\mathrm{BMO}_{w, d, t}} \int \frac{w(x)}{(1+|x|)^{n}} d x .
$$

Thus we have

$$
\frac{1}{w(I)} \int_{I}\left|\phi b-(\phi b)_{I}\right| d x \leq C\left(\|b\|_{\mathrm{BMO}_{w}}+\int_{|x| \leq 1}|b(x)| d x\right) \text {. Q.E.D. }
$$




\section{Remarks}

A counterexample to Theorem 1.7 of Bloom [1]. For the sake of simplicity, we consider the one-dimensional case. Let $w(x)=1 /\left(1+|x|^{1 / 2}\right)$ and $\phi(x) \in$ $C^{\infty}(\mathbb{R})$ such that $\phi\left(x-\frac{1}{2}\right)$ is odd, $\phi(x)=1 \quad(x \geq 1)$, and $\|\phi\|_{\infty} \leq 1$. Then one can show that $\phi$ satisfies (1) and (2) in Theorem 1, but $\phi \notin \mathrm{BMO}_{w}$. In fact, for $a>2$

$$
\int_{1-a}^{a}\left|\phi(x)-\phi_{(1-a, a)}\right| d x \geq 2 a-2, \quad-\int_{1-a}^{a} \frac{1}{1+|x|^{1 / 2}} d x \leq C a^{1 / 2} .
$$

This implies $\phi \notin \mathrm{BMO}_{w}$. Next, one sees easily

$$
\int_{I^{c}} \frac{w(x)}{\left|x-x_{I}\right|} d x \leq C|I|^{-1 / 3} \text { for } I: \text { intervals. }
$$

We also have

(i) If $I \cap[0,1]=\varnothing, \int_{I}\left|\phi-\phi_{I}\right| d x=0$.

(ii) If $I \cap[0,1] \neq \varnothing$ and $|I|<4$, then

$$
\int_{I}\left|\phi-\phi_{I}\right| d x \leq C\|\phi\|_{\text {Lip } 1}|I|^{2} .
$$

Also $w(I) \sim|I|$. Hence

$$
\frac{w(I)}{\int_{I^{c}} \frac{w(x)}{\left|x-x_{I}\right|} d x} \geq C|I|^{1+1 / 3} \geq C|I|^{2} \geq C \int_{I}\left|\phi-\phi_{I}\right| d x .
$$

(iii) If $I \cap[0,1] \neq \varnothing$ and $|I| \geq 4$, then

$$
\begin{gathered}
w(I)=\int_{I} \frac{d x}{1+|x|^{1 / 2}} \leq 2+\int_{1}^{|I|} \frac{d t}{\sqrt{t}} \leq 2|I|^{1 / 2}, \\
w(I) \geq \int_{1}^{|I|} \frac{d x}{1+|x|^{1 / 2}} \geq \frac{1}{2} \int_{1}^{|I|} \frac{d t}{\sqrt{t}} \geq|I|^{1 / 2}-1 \geq \frac{|I|^{1 / 2}}{2} \\
\int_{I^{c}} \frac{w(x)}{\left|x-x_{I}\right|} d x=\int_{(2 I)^{c}} \frac{w(x)}{\left|x-x_{I}\right|} d x+\int_{2 I \backslash I} \frac{w(x)}{\left|x-x_{I}\right|} d x \\
\leq C|I|^{-1 / 2}+C|I|^{-1} w(2 I) \leq C|I|^{-1 / 2}
\end{gathered}
$$

Hence

$$
\frac{w(I)}{\int_{I^{c}} \frac{w(x)}{\left|x-x_{I}\right|} d x} \geq C|I| \geq C \int_{I}\left|\phi(x)-\phi_{I}\right| d x .
$$

Therefore, $\phi$ satisfies conditions (1) and (2) for $w(x)=1 /\left(1+|x|^{1 / 2}\right)$.

Remark 1. The assumption $(*)$ is used to prove Propositions 1 and 2. The equivalence of $\|f\|_{H_{w, d}^{1}}$ and $\left\|M_{d}^{*} f\right\|_{L^{\prime}(w)}$ does not hold for general doubling weights. We give an example.

Example 1. For $w(x)=|x-1|, w(x) \in A_{\infty}(\mathbb{R})$ and hence $w(x)$ is a doubling measure. For this $w(x)$ there exists no $C>0$ such that

$$
\left\|M_{d}^{*} a\right\|_{L^{1}(w)} \leq C\|a\|_{H_{w, d}^{1}} \text { for all }(w, d) \text {-atoms } a \text {. }
$$


In fact, for $j \in \mathbb{N}$ let

$$
\begin{aligned}
a_{j}(x) & =2^{j}\left(\chi_{\left[1-2^{-j+1}, 1-2^{-j}\right)}-\chi_{\left[1+2^{-j}, 1+2^{-j+1}\right)}\right), \\
\varphi_{j}^{+}(x) & =2^{j+1} \chi_{\left[1+2^{-j-1}, 1+2^{-j}\right)}-2^{j} \chi_{\left[1+2^{-j}, 1+2^{-j+1}\right)}, \\
\varphi_{j}^{-}(x) & =-2^{j+1} \chi_{\left[1-2^{-j}, 1-2^{-j-1}\right)}+2^{j} \chi_{\left[1-2^{-j+1}, 1-2^{-j}\right)} .
\end{aligned}
$$

Then $a_{j}^{+}(x)=\frac{\varphi_{j}^{+}(x)}{2^{j+1} w\left(\left[1,1+2^{-j+1}\right)\right)}$ and $a_{j}^{-}(x)=\frac{\varphi_{j}^{-}(x)}{2^{j+1} w\left(\left[1-2^{-j+1}, 1\right)\right)}$ are $(w, d)$ atoms. Let $\lambda_{j}=2^{j+1} w\left(\left[1,1+2^{-j+1}\right)\right)=2^{j+1} w\left(\left[1-2^{-j+1}, 1\right)\right)$. Then

$$
\begin{gathered}
\sum_{k=j}^{\infty} \lambda_{k} \leq \sum_{k=j}^{\infty} 2^{k+1} \int_{0}^{2^{-k+1}} t d t=\frac{2^{2-j}}{\left(1-2^{-1}\right)} \\
a_{j}(x)=\sum_{k=j}^{\infty} \lambda_{k} a_{k}^{+}(x)+\sum_{k=j}^{\infty} \lambda_{k} a_{k}^{-}(x) \text { in } \mathscr{S}^{\prime}(\mathbb{R})\left(\text { and in } L^{1}(w)\right) .
\end{gathered}
$$

Hence $\left\|a_{j}\right\|_{H_{w, d}^{1}} \leq C 2^{-j}$. On the other hand, for $x \in[0,1)$ we have $M_{d}^{*} a_{j}(x) \geq\left|\int_{0}^{1} a_{j}(t) d t\right|=1$, and so $\int_{-\infty}^{\infty} M_{d}^{*} a_{j}(x) w(x) d x \geq \int_{0}^{1} w(x) d x=\frac{1}{2}$. Since $2^{-j+1} a_{j}(x) / w([0,2))$ is a $(w, d)$-atom, these show our assertion.

Remark 2. E. Nakai characterizes the pointwise multipliers of $\mathrm{BMO}_{w}$ in the case $w(I(x, s r)) \leq C s^{n+1} w(I(x, r)), s \geq 1, x \in \mathbb{R}^{n}, r>0$ (where $I(x, r)$ is the cube with center $x$ and sidelength $r$, this condition is called $D_{n+1}$ in [5]), including the case $\int \frac{w(x) d x}{1+|x|^{n}}=+\infty$. He also considers more general BMO spaces than ours. By our method, the converse of Proposition 3 can be derived under the condition $w \in D_{n+1}$, and hence is included in the work of Nakai.

However, there are doubling weights that satisfy $(*)$ and $\int \frac{w(x) d x}{1+|x|^{n}}<\infty$, but does not satisfy $D_{n+1}$. We give an example in the one-dimensional case, for the sake of simplicity, however, the proof we omit.

Example 2. Let $1<r<+\infty$ and $0<\alpha<\beta<1$. Set

$$
w(x)= \begin{cases}\min \left(|x|^{-\alpha},\left(2^{-j \beta /(r-1)}+\left|x-2^{j}\right|\right)^{r-1}\right), & \left|x-2^{j}\right|<1, \\ |x|^{-\alpha}, & \text { otherwise. }\end{cases}
$$

Then, $w \in A_{p} \quad(p>r)$ but $w \notin A_{r}, w \notin D_{s} \quad(s<r)$. It also holds that $w(x) \geq|x|^{-\beta}$ and $\int \frac{w(x) d x}{1+|x|}<+\infty$.

Remark 3. Multiplication operators are not well defined on cosets of BMO. So, BMO should be a set of functions, when one says " $\phi$ multiplies BMO", etc. In this context, even in the unweighted case the set of pointwise multipliers of $\mathrm{BMO}\left(\mathbb{R}^{\mathrm{n}}\right)$ contains nonconstant functions (cf. [4]), which contradicts Bloom's Theorem 1.6. Although we cannot trust his theorems concerning $\mathrm{BMO}_{w}$ in [1], his ideas are good ones. Our work depends heavily on his paper.

The author thanks the referee for his suggestions, especially in Proposition 3 and Remark 3. 


\section{REFERENCES}

1. S. Bloom, Pointwise multipliers of weighted BMO spaces, Proc. Amer. Math. Soc. 105 (1989), 950-960.

2. R. R. Coifman and G. Weiss, Extensions of Hardy spaces and their use in analysis, Bull. Amer. Math. Soc. 83 (1977), 569-645.

3. E. Nakai, Pointwise multipliers for functions of weighted bounded mean oscillation, Studia Math. (to appear).

4. E. Nakai and K. Yabuta, Pointwise multipliers for functions of bounded mean oscillation, J. Math. Soc. Japan 37 (1985), 207-218.

5. J.-O. Strömberg and A. Torchinsky, Weighted Hardy spaces, Lecture Notes in Math., vol. 1381, Springer-Verlag, Berlin and Heidelberg, 1989.

6. A. Uchiyama, Lecture notes (285I), Department of Mathematics, University of California, Los Angeles, 1980-1981.

Department of Mathematics, Nara Women's University, Kita-Uoya Nishimachi, Nara 630, JAPAN 